\title{
HIGHLY SEGMENTED LARGE-AREA HYBRID PHOTODIODES WITH BIALKALI PHOTOCATHODES AND ENCLOSED VLSI READOUT ELECTRONICS
}

\author{
A. Braem, E. Chesi, F. Filthaut, A. Go, C. Joram* P. Weilhammer, P. Wicht \\ CERN, EP Division, Geneva, Switzerland
}

\author{
W. Dulinski \\ LEPSI, Strasbourg, France \\ J. Séguinot \\ Collège de France, Paris, France \\ H. Wenzel \\ Phototube consultant, San Ramon, CA, U.S.A. \\ T. Ypsilantis \\ INFN Bologna, Italy
}

\begin{abstract}
We report on the principles, design, fabrication, and operation of a highly segmented, large-area hybrid photodiode, which is being developed in the framework of the LHCb RICH project. The device consists of a cylindrical, $127 \mathrm{~mm}$ diameter vacuum envelope capped with a spherical borosilicate UV-glass entrance window, with an active-to-total-area fraction of $81 \%$. A fountain-focusing electron optics is used to demagnify the image onto a $50 \mathrm{~mm}$ diameter silicon sensor, containing 2048 pads of size $1 \times 1 \mathrm{~mm}^{2}$. The sensor pads are read out by 16 analogue multiplexed readout chips enclosed in the vacuum envelope. A large ultra-high vacuum plant for the evaporation of visible-light transmittive bialkali $\left(\mathrm{K}_{2} \mathrm{CsSb}\right)$ photocathodes and subsequent tube encapsulation has been built and successfully operated. The characteristics and performance of the first HPDs have been measured to be in full agreement with expectations.
\end{abstract}

*Corresponding author. E-mail Christian.Joram@cern.ch. 


\section{Introduction}

Since the BEAUNE 96 conference [1], major progress has been made in the development of Hybrid Photodiodes (HPD). Highly segmented, large-area HPDs have been developed which integrate the readout electronics in the vacuum envelope in order to reduce the number of vacuum feedthroughs needed for the photodetector readout.

At BEAUNE 96, two HPD developments at CERN were presented [2,3], which were still in their first stages. Both developments are carried out in the framework of the Ring Imaging Cherenkov ( $\mathrm{RICH})$ detector readout of the $\mathrm{LHCb}$ experiment [4].

The development of HPDs constitutes a major advance for the RICH technique because of their

- high granularity, i.e. very large number of pixels (up to 2000), combined with a high spatial resolution;

- sensitivity to visible light, which simplifies the detector construction and results in better performances (merit factor $N_{0}$, chromatic aberrations) compared to UV gas detectors;

- excellent energy resolution compared to photomultiplier tubes;

- high rate capability.

These properties make HPDs suitable also for other applications, such as calorimeter readout, fibre tracking or medical imaging [5].

\section{The Pad HPD}

\subsection{The basic design concept}

The design of our HPD, which in the following will be called Pad HPD, is oriented to the requirements of the readout of the LHCb RICH counters.

An area of about $2.9 \mathrm{~m}^{2}$ has to be equipped with photodetectors providing high sensitivity (visible and near UV range), single photon detection, a granularity of about $2.5 \times 2.5 \mathrm{~mm}^{2}$, operation at the LHC bunch crossing rate of $40 \mathrm{MHz}$, as well as a maximum active area coverage. The resulting high number of readout channels (about $4 \times 10^{5}$ ) demand the per-channel-cost to be kept below about $10 \mathrm{CHF}$.

The Pad HPD is a round photodetector of $127 \mathrm{~mm}$ (5 inch) diameter (see Fig. 1), with a $114 \mathrm{~mm}$ diameter active area visible-light transmissive bialkali photocathode. Hexagonal close packing of the HPDs results in an active area coverage of over $70 \%$. The spherical entrance window of the HPD is made of an UV extended borosilicate glass ( $\mathrm{T}=50 \%$ at $\lambda=250 \mathrm{~nm}$ ). The window and the 

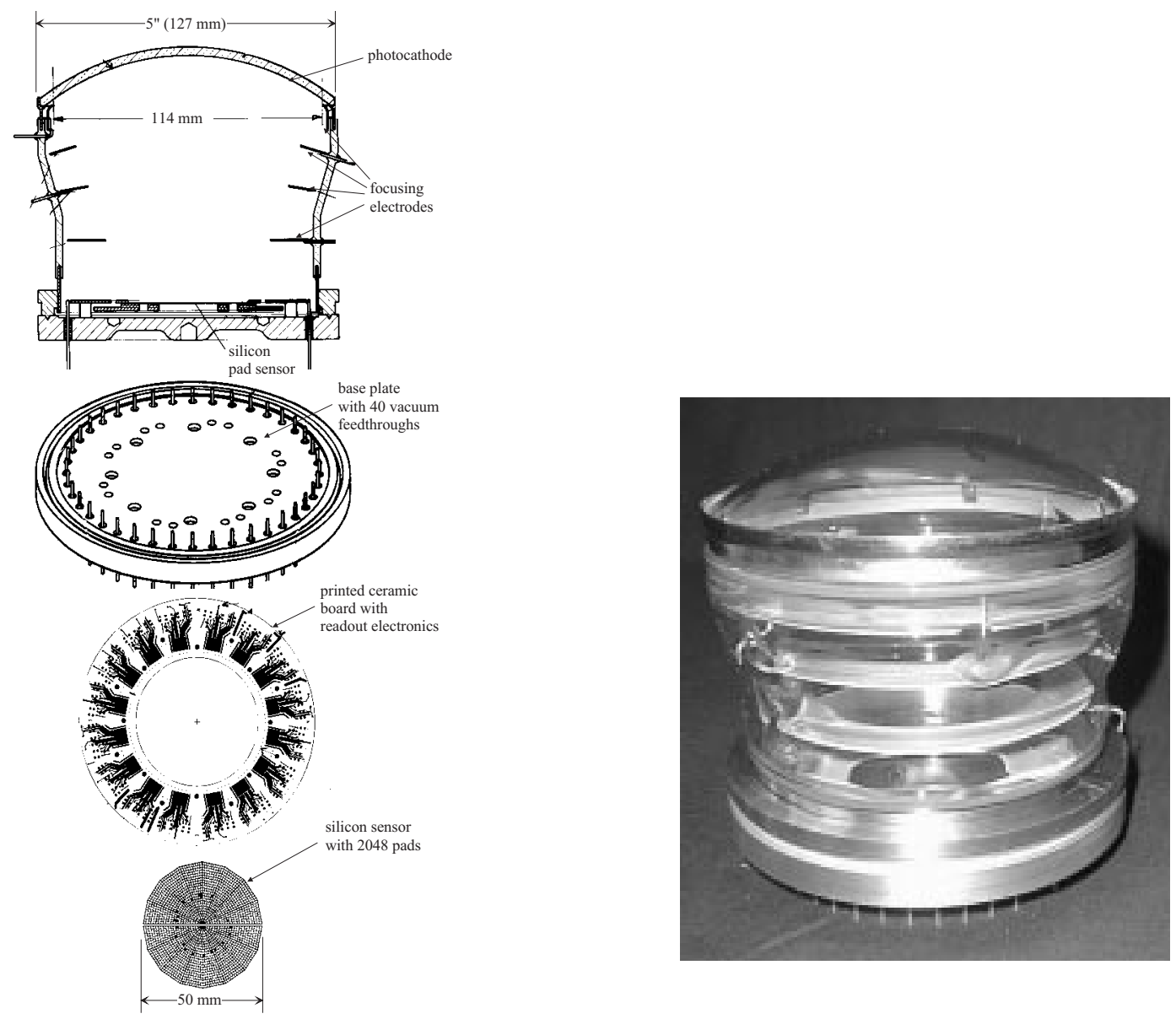

Figure 1: Longitudinal-sectional view and photograph of a sealed Pad HPD with integrated readout electronics.

tube body* are joined by a Kovar skirt in order to adapt to the slightly different thermal expansion coefficients of the glass types. A set of 4 stainless steel ring electrodes defines a fountain shape electrostatic configuration, which demagnifies the photocathode image by a factor $\approx 2.5$ onto a silicon sensor of $50 \mathrm{~mm}$ diameter. The sensor comprises 2048 pads of size $1 \times 1 \mathrm{~mm}^{2}$ and is read out by multiplexed analogue electronics enclosed in the vacuum envelope. The resulting readout granularity at the HPD entrance window matches the LHCb requirements. Both the sensor and the electronic chips are mounted on a ceramic carrier, which is wire bonded to the 40 vacuum feedthroughs of the stainless steel base plate.

The design of the Pad HPD is also strongly motivated by the chosen fabrication process (see chapter 3), which consists of an external photocathode evaporation followed by an insitu cold

\footnotetext{
* The vacuum envelope, focusing electrodes and the base plate are fabricated by SVT, F-91170 Viry Chatillon, France.
} 
indium sealing of the base plate to the HPD envelope.

For comparison, the second development project undertaken at CERN aims to produce, in collaboration with industry ${ }^{\dagger}$, an $80 \mathrm{~mm}$ diameter (sensitive area diameter: $72 \mathrm{~mm}$ ), cross-focused HPD [6] containing 1024 measurement pixels read out using binary electronics.

\section{$2.2 \quad$ Electron optics}

The electrostatic configuration of the Pad HPD is defined in the following way: The electric field between the photocathode $\left(\mathrm{U}_{C}=-20 \mathrm{kV}\right)$ and the silicon sensor plane $(\mathrm{U}=0 \mathrm{~V})$ is shaped by 4 concentric ring electrodes such that the photocathode image is linearly demagnified onto the silicon sensor. The second electrode allows control of the demagnification. The first electrode (the so-called bleeder electrode) corrects nonlinearities and cross-focusing effects at the edge of the cathode. Figure 2 shows electron trajectories in the Pad HPD simulated with the code SIMION[7].

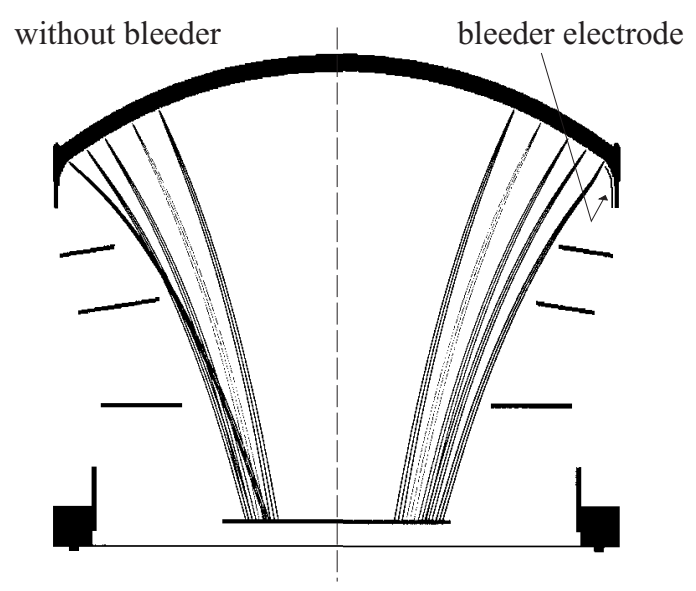

Figure 2: Simulated electron trajectories. Left: Crossing trajectories in a tube without bleeder electrode. Right: Trajectories in a tube with bleeder electrode. For each emission point, photoelectrons with an initial kinetic energy of $1 \mathrm{eV}$ and emission angles of $0^{\circ}$ and $\pm 45^{\circ}$ are shown.

\subsection{The silicon sensor}

The sensor consists of a $300 \mu \mathrm{m}$ thick round silicon disk of $52 \mathrm{~mm}$ (50 mm active) diameter ${ }^{\ddagger} .2048$ pads of $980 \mu \mathrm{m} \times 980 \mu \mathrm{m}$ size are implemented as $\mathrm{p}^{+} \mathrm{n}$ junctions on the high resistive bulk $(5 \mathrm{k} \Omega \cdot \mathrm{cm})$.

\footnotetext{
†Delft Electronic Products, Rhoden, The Netherlands.

¥The silicon sensors have been processed at CSEM, CH-2007 Neuchâtel, Switzerland, or at SINTEF, Trondheim, Norway.
} 
The spacing between the $\mathrm{p}^{+}$implants is $40 \mu \mathrm{m}$. The pads are arranged in 16 sectors in a nearly circular geometry. They are capacitively coupled to readout lines, which are routed on top of a $4 \mu \mathrm{m}$ thick $\mathrm{SiO}_{2}$ insulator to the bonding pads, and are placed at the circumference of the sensor. In the Pad HPD the accelerated photoelectrons impinge on the silicon sensor from the back side. Therefore special care has been taken to minimise the energy loss in the aluminium contact and the nondepleted $\mathrm{n}^{+}$layer.

\subsection{The readout electronics}

The Pad HPD is equipped with a low-noise analogue readout chain. An analogue scheme is believed to be crucial for the robust readout of small signals at LHC speed. Compared to a binary readout, lower noise figures can be obtained because disturbances, due to the switching of a comparator, are avoided. The availability of the signal amplitudes provides an efficient and direct way to monitor online the performance of the detector and the readout chain. Instabilities, like gain and noise variations or common mode noise effects, can be handled by continuously updating the pedestal average and variance using Digital Signal Processors (DSP). The analogue readout exploits the excellent energy resolution which is one of the most attractive features of the HPD principle and which can be used to recognize multiple photoelectrons in the pattern recognition in high density RICH images.

To realise a HPD with a high number of readout channels, the readout electronics is enclosed in the vacuum envelope. The number of vacuum feedthroughs is kept at a technically feasible number by signal multiplexing. Apart from a low power consumption, compatibility with typical ultra-high vacuum bake-out cycles and negligible long term outgassing are indispensable. The fact that the electronics can be positioned very close to the Si sensor represents a major advantage in view of the very small signal of less than $1 \mathrm{fC}$ created by single photoelectrons in the sensor. Minimal capacity at the amplifier input is essential for the lowest possible noise and a stable operation.

The measurements presented in this paper have been obtained with the Viking VA3 electronics [8]. The VLSI chip comprises, for each of its 128 channels, a front-end charge amplifier, a shaper, and a sample-and-hold unit, which determines the maximum amplitude of the shaped signal. The power consumption is $\approx 300 \mu \mathrm{W} /$ channel. The VA3 with a shaping time of $1.3 \mu$ s is clearly too slow to be used at LHC. Therefore, a low capacity (LC) version of the analogue SCTA128 chip [9] has been developed, which will be used in the final Pad HPD. The chip provides signal shaping with a peaking time of $25 \mathrm{~ns}$ and also incorporates the LHC-specific features like a 128 cell deep analogue pipeline, a derandomising buffer (8 cells deep) and $40 \mathrm{MHz}$ analogue multiplexing. Tests with the recently available SCTA128LC chip demonstrated the design peaking time and the fast multiplexing. From measurements where the SCTA128LC was connected to a small silicon sensor, 
pedestal noise values as low as 600 to $700 \mathrm{e}^{-}$are expected when connected to a Pad HPD sensor. The integration of the SCTA128LC in the Pad HPD is foreseen in the next months.

\section{Fabrication of the Pad HPD}

The Pad HPD fabrication uses an external photocathode evaporation process (sometimes referred to as transfer technique) followed by an insitu encapsulation of the tube with the base plate, using a cold indium sealing technique. The silicon sensor and the readout electronics are mounted on the base plate prior to the evaporation process. During the cathode processing, the base plate is kept at a safe distance from the envelope. This technique was chosen:

- to avoid a destructive contamination of the silicon sensor and the readout electronics by the alkali metal vapours (K, Cs) during the evaporation process;

- to minimise the thermal load on the sensor and electronics during the bakeout procedure and the sealing, which could lead to irreversible damage to the semiconductor material.

A further advantage of the technique is the possibility to reopen sealed tubes, which do not operate with a satisfactory performance. It has been demonstrated that all HPD components, in particular the relatively expensive silicon sensor and electronics chips, can be reused after such an intervention.

\subsection{Photocathode processing}

In the literature various processes are described to produce semi-transparent bialkali ( $\left.\mathrm{K}_{2} \mathrm{CsSb}\right)$ photocathodes. The processes can be divided into two classes: Sequential processes, where antimony and then the alkali metals are evaporated one after the other, and co-evaporation processes, where usually Sb and K are evaporated simultaneously. Numerous variants exist, which differ mainly in the number and order of the steps and the substrate temperature cycle. The details of a process depend on many parameters (e.g., geometrical configuration of the substrate and the evaporation sources, temperature gradients, pumping conductance) and have always to be determined by iterative optimisation. We have adopted a co-evaporation process which comprises about 20 individual evaporation steps. In comparison to the simpler sequential processes, co-evaporation permits a reduction in the amount of evaporated materials. This reduces the contamination of the tube with alkali films which may lead to a degradation of the high voltage capability of the sealed tube.

The Pad HPD is fabricated in a large ultra-high vacuum plant which has been designed and built at CERN [10]. After a bake-out at $160^{\circ} \mathrm{C}$, the plant reaches ultimate pressures of a few $10^{-10}$ mbar. The pressure at the start of the photocathode processing, when the vacuum tank is kept at an elevated temperature, is typically $2 \times 10^{-9} \mathrm{mbar}$. The composition of the residual gas is 
monitored by means of a quadrupole mass spectrometer. Care is taken to keep the partial pressures of water and any other reactive substances $\left(\mathrm{O}_{2}, \mathrm{CO}\right)$ below $1 \times 10^{-9}$ mbar. The residual gas consists to about $90 \%$ of hydrogen which is outgassing from the stainless steel walls. An internal tubular heating spiral is used to bake out the $\mathrm{HPD}$ envelope at a temperature of about $300^{\circ} \mathrm{C}$, which is necessary to fully remove water also from the bulk of the tube envelope. The evaporation plant is controlled by a distributed slow control system under PC control ${ }^{\S}$.

The envelope is mounted on a rigid support structure in the centre of the tank. A transfer system allows a carriage which is equipped with the three evaporation sources to be positioned such that the sources sit close to the centre of curvature of the spherical tube window. During the processing, the envelope is kept at a constant temperature of $150^{\circ} \mathrm{C}$.

The evolution of the photocathode sensitivity is monitored online by measuring the photocurrent between the photocathode and the focusing ring electrodes (anode), where a collection voltage of typically $100 \mathrm{~V}$ is applied. The cathode is exposed to white or monochromatic light of precisely known intensity. Knowledge of the true photocurrent, i.e., the quantum efficiency, and possible background currents, is essential for the optimisation of the photocathode.

\subsection{Tube encapsulation}

A cold indium sealing technique has been developed to encapsulate the envelope with the base plate. The method requires a number of preparatory steps. An indium wire of $99.99 \%$ purity is placed in the circular V-shaped groove on the base plate. A $10 \mu \mathrm{m}$ thick nickel layer was galvanically deposited on the groove surface to improve the wetting and uniformity of the indium. Heating the baseplate in a dedicated set-up under vacuum at $250^{\circ} \mathrm{C}$ melts the indium. The liquid indium is stirred under vacuum by means of a rotating needle in order to completely degas it and further improve its uniformity. The contact surface of the envelope, i.e., the knife edge of the envelope's flange, is deposited with a $10 \mu \mathrm{m}$ thick nickel layer and an evaporated gold film of $500 \mathrm{~nm}$ thickness.

Once the pretreatment is finished, and the silicon sensor and the electronics are mounted and wire bonded to the vacuum feedthroughs on the base plate, the base plate is installed in the photocathode evaporation plant on a second movable carriage. The tube encapsulation is performed several hours after the photocathode processing, when the cathode has cooled down to room temperature. The carriage is then aligned under the envelope. A press mechanism allows the base plate to be pushed gradually against the flange of the envelope with a force of $25 \mathrm{kN}$. The knife edge cuts into the indium filled groove. The high mechanical pressure associated with this technique results in perfectly tight vacuum seals, which do not require additional clamping.

§Fieldpoint I/O system, BridgeView software, National Instruments 


\section{Performance}

Numerous measurements characterising the HPD have been performed. We present quantum efficiency measurements, which have been obtained online during the photocathode evaporation, and subsequently a number of measurements, where the sealed HPD has been exposed to a low intensity $\mathrm{H}_{2}$ flash lamp. The narrow spot of the flash lamp $(\sigma \approx 1 \mathrm{~mm})$ can be precisely scanned over the HPD window by means of a movable mirror.

\subsection{Quantum efficiency of the $\mathrm{K}_{2} \mathrm{CsSb}$ photocathodes}

The quantum efficiency is measured online during the photocathode evaporation. Light from a tungsten halogen lamp is focused through a viewport in the vacuum tank onto the HPD entrance window. A set of interference filters (combined with appropriate broad band pass filters) provides a selection of wavelengths between $308 \mathrm{~nm}(4 \mathrm{eV})$ and $651 \mathrm{~nm}(1.9 \mathrm{eV})$. The intensity of each individual wavelength is determined by means of a calibrated photodiode. The light is chopped by an electronic shutter so that any background can be subtracted. The quantum efficiency is then measured in DC current mode by applying a voltage of typically $100 \mathrm{~V}$ between the photocathode and the focusing ring electrodes. Figure 3, left, shows the quantum efficiency of a recently produced HPD. It reaches a maximum of about $20 \%$ around $350 \mathrm{~nm}$. All recently produced cathodes had peak quantum efficiencies between $15 \%$ and $20 \%$. The further optimisation of the quantum efficiency is the subject of current studies. The light spot, which has a size of about $2 \mathrm{~cm}^{2}$ can be scanned over the window surface. The uniformity of the efficiency is typically better than $\pm 10 \%$, where part of it may be due to a nonuniform and incomplete collection efficiency with the field configuration used. A clarification of this point will follow from measurements with a Cherenkov radiator. Devices which had been subject to correct baking of the vacuum plant and the HPD components, have shown no measurable degradation of the quantum efficiency over time scales of months.

\subsection{Electron optical parameters}

Although the design value for the tube high voltage is $20 \mathrm{kV}$, the detector has been stably operated at up to $30 \mathrm{kV}$. The fountain shape field configuration is able to produce an almost purely linear imaging over the full active diameter $(114 \mathrm{~mm})$. The demagnification factor can be adjusted by varying the voltage of the second electrode. Figure 3, right, shows the correlation between the radial coordinate of the light spot on the photocathode and the centre of gravity of the charge distribution on the silicon sensor. The slope of a linear curve fitted to the data gives a demagnification of 2.7. The fit residuals of typically $200 \mu \mathrm{m}$ can be reduced to about $50 \mu \mathrm{m}$ by allowing a superposition of two linear curves. 

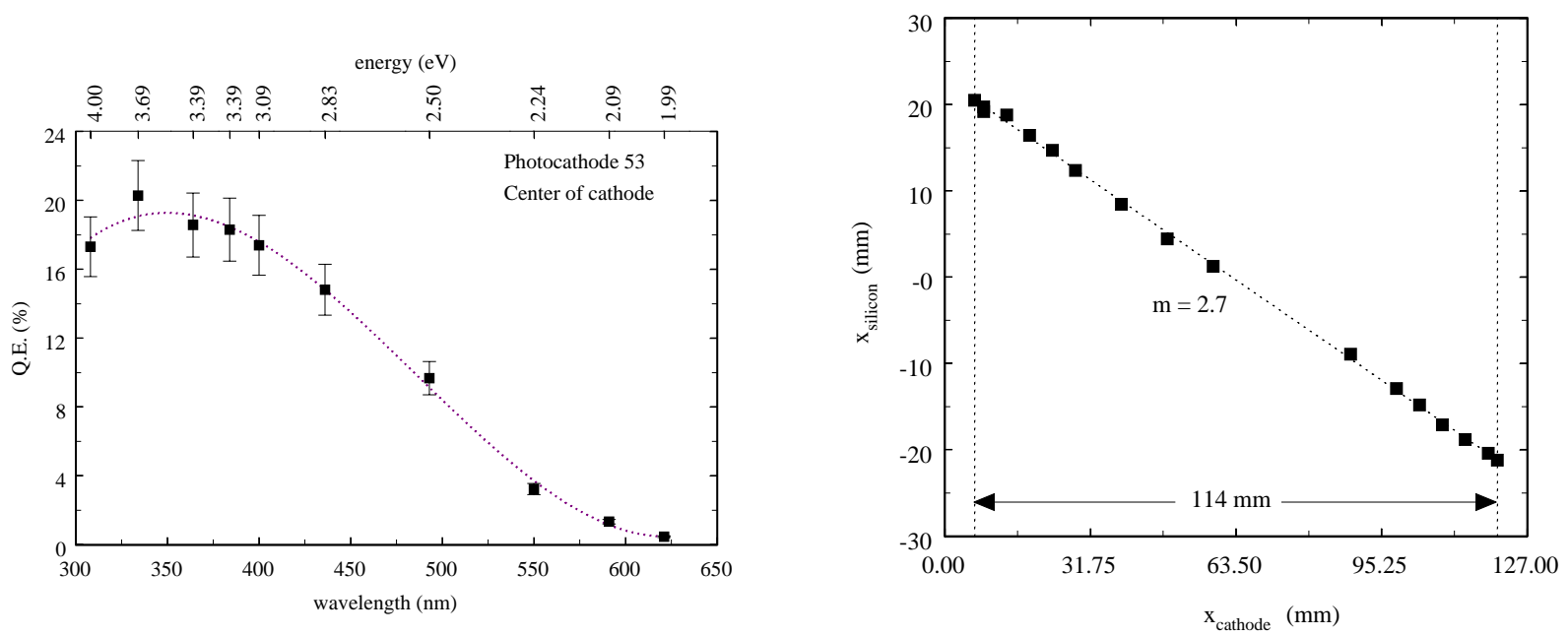

Figure 3: Left: Quantum efficiency versus wavelength of a recently produced HPD. Right: Correlation between the photon detection point and the centre of gravity of the charge distribution measured on the silicon sensor.

The point spread function, i.e., the spatial distribution of the photoelectrons on the silicon anode originating from a point-like spot on the photocathode, is difficult to determine with our current set-up, because of the comparably large light spot and (for this purpose) the relatively coarse granularity of the silicon sensor. From fitting the charge distribution of single photoelectrons on the silicon sensor and unfolding the contributions of the spot size and the granularity of the sensor, for $20 \mathrm{kV}$ a point spread function with a Gaussian width of $400 \mu \mathrm{m}$ is found. This is to compared with the pixelization error $\sigma_{\text {pixel }}=1 \mathrm{~mm} / \sqrt{12}=288 \mu \mathrm{m}$. For voltages above $23 \mathrm{kV}$, the point spread contribution is less than the pixel error.

\subsection{Signal characteristics}

An HPD provides perfect linearity between the signal amplitude and the voltage applied between the anode and cathode. Figure 4, left, shows the signal amplitude for single photoelectrons in ADC channels, as a function of the anode-cathode voltage. The intersection with the horizontal axis at $0.53 \mathrm{kV}$ corresponds to an energy loss of only $530 \mathrm{eV}$ in the backplane metallisation and the nondepleted $\mathrm{n}^{+}$layer of the silicon. The ADC calibration follows from the slope of the curve (3.929 ADC bins $/ \mathrm{kV}$ ) and the energy required to create an electron-hole pair (in the following, simply called electrons or $\left.\mathrm{e}^{-}\right)$in silicon $\left(3.62 \mathrm{eV} / \mathrm{e}^{-}\right): 1 \mathrm{ADC}$ bin $=70.3 \mathrm{e}^{-}$. A pulse height spectrum at $20 \mathrm{kV}$ is shown in Fig. 4, right. The pedestal, which has a Gaussian width of $\sigma_{\text {noise }}=4.04$ ADC bins or $285 \mathrm{e}^{-}$, has been subtracted and a $4 \sigma_{\text {noise }}$ cut has been applied. The single photoelectron peak appears at 77.5 ADC counts (5.02 ADC counts width). The signal-to-noise ratio is 19. The 
background in Figure 4, right, is due to the characteristic backscattering of photoelectrons from the silicon surface, which occurs with a probability of about $18 \%$. Assuming a uniform distribution of the deposited energy of back-scattered photoelectrons between 0 and the full energy, the single photoelectron detection efficiency is expected to be $94 \%$.
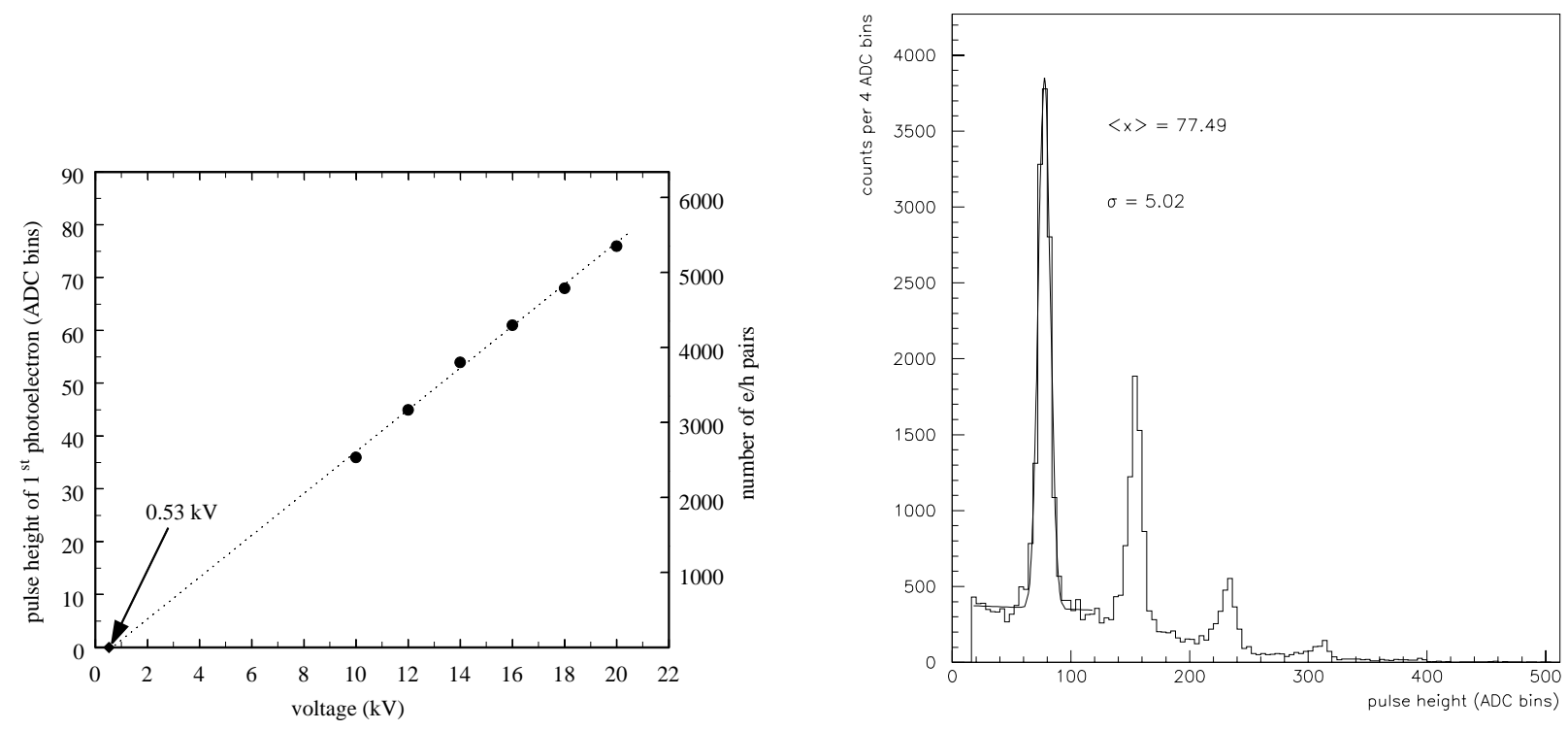

Figure 4: Left: Measured signal amplitude for single photo-electrons versus anode-cathode voltage. Right: Measured pulse height spectrum in ADC counts at $20 \mathrm{kV}$ for an average number of photoelectrons of 0.9. The pedestal has been subtracted and a $4 \sigma_{\text {noise }}$ cut has been applied.

\section{$5 \quad$ Status and outlook}

The first sealed HPDs with bialkali photocathodes and integrated readout electronics have been fabricated and successfully tested. The performance of the detectors is in good agreement with the design expectations and makes it an excellent candidate for the LHCb RICH detector readout. Whereas the past year has been mainly devoted to developing the complex production technologies, we are now in the phase of fine tuning the various parameters, in order to further optimise the quantum efficiency of the photocathode and the production yield. In parallel, preparatory work is going on in order to fabricate HPDs with the fast SCTA128 electronics still in 1999. A test of the HPD in a Cherenkov radiator set-up, which is scheduled for July 1999, will be used to further characterise the performance of the detector. 


\section{Acknowledgements}

We would like to thank M. Alemi and D. Ferenc for carrying out the electron trajectory simulations. The competent advice from G. Lion on chemical questions is gratefully appreciated. We would like to thank D. Fraissard and P. Carrie for their efficient technical support, and thank R. Boulter for mounting and bonding the sensors and the electronic chips. We acknowledge the partial support for this work from the CERN-LAA project.

\section{References}

[1] R. DeSalvo, Nucl. Instr. Meth. A387 (1997) 92.

[2] E. Chesi et al., Nucl. Instr. Meth. A387 (1997) 122.

[3] T. Gys et al., Nucl. Instr. Meth. A387 (1997) 131.

[4] LHCb Technical Proposal, CERN/LHCC 98-4, LHCC/P4, 1998.

[5] C. Joram, Proceeding of the VIth Int. Conf. on Advanced Technology and Particle Physics, Como, Italy, 1998, in press.

[6] M. Alemi et al., Proceeding of the 1998 Nuclear Science Symposium and Medical Imaging Conference, Toronto, Canada, 1998, in press.

[7] D. Ferenc, Proceeding of the 1998 Nuclear Science Symposium and Medical Imaging Conference, Toronto, Canada, 1998, in press.

[8] P. Weilhammer et al., Nucl. Instr. Meth. A384 (1996) 159.

[9] F. Anghinolfi et al., IEEE Transactions Nucl. Sci., Vol. 44, No 3 (1997) 298.

[10] E. Chesi et al., LHCb note, 98-007 RICH. 